BULL. AUSTRAL. MATH. SOC.

VOL. 25 (1982), 215-219.

\title{
ON LOCALLY WEAKLY ALMOST PERIODIC TRANSFORMATION GROUPS
}

\author{
SABER ELAyd I
}

\begin{abstract}
It is shown that a transformation group with a locally compact Hausdorff phase space and an. abelian phase group is locally weakly almost periodic if and only if it is P-locally weakly almost periodic for some replete semigroup $P$ in the phase group.
\end{abstract}

Let $(X, T, \pi)$ be a transformation group, where $X$ is assumed to be a locally compact Hausdorff space and $T$ is an abelian topological group. In [1] Coven showed that, for several dynamical properties the phase space $X$ possesses the property under $T$ if $X$ possesses the property under some replete semigroup $P$ in $T$. This was shown for the properties of almost periodicity, regular almost periodicity and isochronousity. The main purpose of this note is to extend Coven's results to the property of locally weakly almost periodicity. We use an entirely new technique in the theory of topological dynamics; namely the so called prolongational technique. The prolongation notions were originally introduced by Ura [5] for dynamical systems, then were generalized in the setting of transformation groups by Hajek [4]. The author [2] developed and used these techniques in a more general form.

For the convenience of the reader we give the definition of the basic notions used in this note. For $x \in X$, and a replete semigroup [3] $P$ in $T$, we have the following definitions:

Received 29 September 1981. 
the P-limit set of $x$ :

$$
\left.L ^ { P } ( x ) = n \longdiv { x p P } \mid p \in P\right\}
$$

the P-prolongation set of $x$ :

$$
D^{P}(x)=n\{\sqrt[V P]{V} \mid V \text { is a neighbourhood of } x\} .
$$

Note that one can extend the transformation group $(X, T, \pi)$ to $\left(X^{*}, T, \pi^{*}\right)$, where $X^{*}=X \cup\{\infty\}$ is the one point compactification of $X$, by letting $\pi^{*}(x, t)=(x, t)$ for each $x \in X$ and $t \in T$ and $\pi^{*}(\infty, t)=\infty$ for each $t \in T$. The corresponding notions in $\left(X^{*}, T, \pi^{*}\right)$, of $L^{P}(x)$ and $D^{P}(x)$ are denoted, respectively, by $L_{*}^{P}(x)$ and $D_{*}^{P}(x)$. The closure of a set $A$ in $X^{*}$ is denoted by $\bar{A}^{*}$. The boundary of $A$ is denoted by $\partial A$.

For breviety $\pi(x, t)$ is denoted by $x t$ and $(X, T, \pi)$ is written as $(X, T)$. Our main references for notation and terminology are [2] and [3].

DEFINITION. Let $x \in X$ and let $P$ be a replete semigroup in $T$. A point $x \in X$ is said to be $\{P-10 c a l l y$ weakly almost periodic $\{$ llocally weakly almost periodic\} if whenever $U$ is a neighbourhood of $x$ there exists a neighbourhood, $V$ of $x$ and a compact subset $K$ of $\{P\}\{T\}$ such that $\{V P \subset U K\}\{V T \subset U K\}$. A point $x \in X$ is said to be discretely $\{P-10 c a l l y$ weakly almost periodic\} \{locally weakly almost periodic\} if the set $K$ in the above definition is replaced by a finite subset $F$ of $\{P\}\{T\}$.

A transformation group $(X, T)$ is said to have the property if each point in $X$ has the property.

LEMMA. If a transformation group $(X, T)$ is P-locally weakly almost periodic, then it is discretely P-locally weakly almost periodic.

Proof. Assume that $x \in X$ is P-locally weakly almost periodic. Then there exists a neighbourhood $V$ of $x$ and a compact subset $K$ of $P$ such that $V P \subset U K$. Let $W$ be a neighbourhood of $x$ such that $\bar{W} \subset V$ and $\bar{W}$ is compact. We will show that $\overline{W P}$ is compact. For each $y \in \bar{W}$ choose a compact neighbourhood $U(y)$, a neighbourhood $V(y)$ of $y$ and a compact subset $K(y)$ of $P$ such that $V(y) P \subset U(y) K(y)$. Since $\bar{W}$ is 
compact, $\bar{W} \subset \bigcup_{i=1}^{n} V\left(y_{i}\right)$ for some points $y_{i} \in \bar{W}$. This implies that $\bar{W} P \subset \bigcup_{i=1}^{n} V\left(y_{i}\right) P \subset \bigcup_{i=1}^{n} U\left(y_{i}\right) K\left(y_{i}\right)$

Hence $\overline{\bar{W} P}=\overline{W P} \subset \bigcup_{i=1}^{n} U\left(y_{i}\right) K\left(y_{i}\right)$. Since for each $i, U\left(y_{i}\right) K\left(y_{i}\right)$ is compact, it follows that $\overline{W P}$ is compact. Since $\overline{W P} \subset U K$ and $\overline{W P}$ is compact, $\overline{W P} \subset U F$, for some finite subset $F$ of $P$. Hence $x$ is descretely $P$-locally weakly almost periodic.

THEOREM. A transformation group $(X, T)$ is locally weakly almost periodic if and only if it is P-locally weakly almost periodic.

Proof. Sufficiency. Assume that $(X, T)$ is $P$-locally weakly almost periodic. The proof consists of two parts.

Part 1. We will show that for $x \in X, D(x)=D_{*}(x)=\overline{x T}=\overline{x T}^{*}$. Let $x \in X$ and let $y \in D_{*}^{P}(x)$. Then there are nets $\left\{x_{i}\right\}$ in $X$ and $\left\{p_{i}\right\}$ in $P$ such that $\lim x_{i}=x$ and $\lim x_{i} p_{i}=y$. Let $U \subset X$ be a neighbourhood of $X$. Then by the preceding lemma there exists a neighbourhood $V \subset X$ of $x$ and a finite subset $F$ of $P$ such that $V P \subset U F$. Since $U F$ is a compact subset of $X$, it follows that $y \neq \infty$. Hence $D_{*}^{P}(x)=D^{P}(x)$. We may assume, without loss of generality, that $\left\{x_{i}\right\} \subset V$. Thus the net $\left\{x_{i} p_{i}\right\} \subset V P \subset U F$. This implies that the net $\left\{x_{i} p_{i}\right\}$ is frequently in $U s$ for some $s \in E$. It follows that $y \in U s$ and thus $y s^{-1} \in U$. Let $\left\{U_{i}\right\}$ be a neighbourhood filter of $x$ which is partially ordered by inclusion. Then for each $i$, there exists $s_{i} \in P$ such that $y s_{i}^{-1} \in U_{i}$. Consequently, $\lim y s_{i}^{-1}=x$. Thus $x \in \overline{y T}$. Recall that if $x$ is $P$-locally weakly almost periodic, then $x$ is $P$-almost periodic. Then according to $[1,2.5]$ the point $x$ is discretely aimost periodic. This implies that $\overline{x T}$ is compact and minimal [3]. Furthermore, $\overline{x T}=\overline{x P}$. Hence $y \in \overline{x P}$. Therefore $D_{*}^{P}(x) \subset \overline{x P}$. Since it is always true that $\overline{x P} \subset D_{*}^{P}(x)$, it follows that 
$D_{*}^{P}(x)=D^{P}(x)=\overline{x P}=\overline{x \bar{P}^{*}}$.

Now let $z \in D_{*}(x)$. Then there are nets $\left\{z_{i}\right\}$ in $X$ and $\left\{t_{i}\right\}$ in $T$ such that $\lim z_{i}=x$ and $\lim z_{i} t_{i}=z$. For each $i$, $z_{i} t_{i} \epsilon \overline{z_{i} \bar{T}^{*}}=\overline{z_{i} \bar{P}}=D_{*}^{P}\left(z_{i}\right)=D^{P}\left(z_{i}\right)$. Let $W \subset X$ be a neighbourhood of $x$. Then $z_{i} \in W$ for all $i \geq i_{0}$. Hence $D_{*}^{P}\left(z_{i}\right) \subset \overline{W P^{*}}$ for $i \geq i_{0}$. This implies that $z_{i} t_{i} \in \overline{W P}^{*}$ for $i \geq i_{0}$. Hence $z \in \overline{W P}^{*}$.

Consequently, $z \in D_{*}^{P}(x)=D^{P}(x)=\overline{x P}=\overline{x T}$. Therefore $D_{*}(x)=D(x) \subset \overline{x T}$. Thus $D_{*}(x)=\overline{x T}=D(x)$ for each $x \in X$.

Part 2. Let $x \in X$. We now show that $x$ is locally weakly almost periodic. Let $U \subset X$ be a neighbourhood of $x$. Since $\overline{x T}$ is minimal, $\overrightarrow{x T} \subset U T$. Since $\overline{x T}$ is compact, $\overline{x T} \subset U F$ for some finite subset $F$ of $r$. Claim that there exists a neighbourhood $V \subset U$ of $x$ such that $V T \subset U F$. Assume the contrary; that is, there does not exist such a set $V$. Then there are nets $\left\{x_{i}\right\}$ in $U$ and $\left\{t_{i}\right\}$ in $T$ such that $\lim x_{i}=x$ and $x_{i} t_{i} \in X-U F$, for each $i$. Since $\infty \neq D_{*}(x)$ as it was shown in Part 1, we may assume, without loss of generality, that $\lim x_{i} t_{i}=b \in X-U F$. But then $b \in D(x)=\overline{x T} \subset U F$ and thus we have a contradiction.

Therefore $x$ is locally weakly almost periodic. The necessity is clear. The proof of the theorem is now complete.

\section{References}

[1] Ethan M. Coven, "P-recussion and transformation groups having an equicontinuous replete semigroup", Math. Systems Theory 3 (1969), $170-178$.

[2] Saber Elaydi, "On equicontinuous transformation groups", BuZl. Austral. Math. Soc. 24 (1981), 367-372. 
[3] Walter Helbig Gottschalk and Gustav Arnold Hedlund, Topological dynamics (American Mathematical Society Colloquium Publication, 36. American Mathematical Society, Providence, Rhode Island, 1955).

[4] Otomar Hajek, "Prolongations in topological dynamics", Seminar on differential equations and dynamical systems II, 79-89 (Seminar Lectures, University of Maryland, 1969. Lecture Notes in Mathematics, 144. Springer-Verlag, Berlin, Heidelberg, New York, 1970).

[5] Taro Ura, "Sur le courant extérieur à une région invariante. Prolongements d'une caractéristique et l'ordre de stabilité", Funkcial. Ekvac. 2 (1959), 143-200.

\footnotetext{
Department of Mathematics,

Kuwait University,

PO Box 5969,

Kuwait.
} 